Article

\title{
Vector Similarity Measures of Q-Linguistic Neutrosophic Variable Sets and Their Multi-Attribute Decision Making Method
}

\author{
Jun Ye $\mathrm{1}^{1, *(\mathbb{D})}$, Zebo Fang ${ }^{2}$ and Wenhua Cui ${ }^{1}$ \\ 1 Department of Electrical Engineering and Automation, Shaoxing University, 508 Huancheng West Road, \\ Shaoxing 312000, China; wenhuacui@usx.edu.cn \\ 2 Department of Physics, Shaoxing University, 508 Huancheng West Road, Shaoxing 312000, China; \\ csfzb@usx.edu.cn \\ * Correspondence: yejun@usx.edu.cn or yehjun@aliyun.com; Tel.: +86-575-8832-7323
}

Received: 30 September 2018; Accepted: 16 October 2018; Published: 22 October 2018

check for updates

\begin{abstract}
Since language is used for thinking and expressing habits of humans in real life, the linguistic evaluation for an objective thing is expressed easily in linguistic terms/values. However, existing linguistic concepts cannot describe linguistic arguments regarding an evaluated object in two-dimensional universal sets (TDUSs). To describe linguistic neutrosophic arguments in decision making problems regarding TDUSs, this study proposes a Q-linguistic neutrosophic variable set (Q-LNVS) for the first time, which depicts its truth, indeterminacy, and falsity linguistic values independently corresponding to TDUSs, and vector similarity measures of Q-LNVSs. Thereafter, a linguistic neutrosophic multi-attribute decision-making (MADM) approach by using the presented similarity measures, including the cosine, Dice, and Jaccard measures, is developed under Q-linguistic neutrosophic setting. Lastly, the applicability and effectiveness of the presented MADM approach is presented by an illustrative example under Q-linguistic neutrosophic setting.
\end{abstract}

Keywords: Q-linguistic neutrosophic variable set; vector similarity measure; cosine measure; Dice measure; Jaccard measure; decision making

\section{Introduction}

Since language is used for thinking and expressing habits of humans in real life, the linguistic evaluation for an objective thing is expressed easily in linguistic terms/values [1]. Hence, they were applied to linguistic fuzzy reason [1] and linguistic decision-making (DM) problems [2-9]. Because of linguistic uncertainty and hesitancy in the linguistic evaluation for an objective thing, there exist the representations of interval/uncertain linguistic numbers or hesitant linguistic numbers. Hence, on the one hand, interval/uncertain linguistic numbers were proposed and applied to (group) DM problems in uncertain linguistic setting [10-14]. On the other hand, hesitant linguistic variables (LVs) and hesitant uncertain LVs were presented and applied in (group) DM problems in hesitant (uncertain) linguistic setting [15-19]. In addition, a linguistic cubic variable was put forward based on combining an interval LV with a unique LV and used for DM problems in linguistic cubic setting [20,21]. Further, a linguistic cubic hesitant fuzzy number/variable was presented to depict the hybrid information of its uncertain linguistic argument and its hesitant linguistic argument and utilized for DM problems in linguistic cubic hesitant fuzzy setting [22]. By combining a neutrosophic number with language, a neutrosophic linguistic number and some weighted aggregation operators of neutrosophic linguistic numbers [23] were introduced for neutrosophic linguistic number DM problems, and then the similarity measure and expected value of hesitant neutrosophic linguistic numbers [24] were further presented for DM problems with hesitant neutrosophic linguistic numbers. 
In real life environments, the truth, indeterminacy, and falsity linguistic arguments regarding an objective thing are presented in a human's thinking and expressing process and linguistic neutrosophic variables/numbers (LNVs) were presented to depict truth, falsity, and indeterminacy linguistic degrees independently [25]. Then, some aggregation operators of LNVs [25,26], cosine measures of LNVs [27], and correlation coefficients of LNVs [28] were proposed, respectively, for DM problems in LNV setting. Regarding the combination of a neutrosophic linguistic number and an LNV, linguistic neutrosophic uncertain numbers and their weighted aggregation operators were presented for DM in uncertain linguistic setting [29]. By the hybrid form of an interval LV (an uncertain linguistic argument) and a single-valued LNV (an argument of confident degree), single-valued linguistic neutrosophic interval LVs, and their weighted aggregation operators were proposed for DM along with uncertain/interval linguistic arguments and their linguistic neutrosophic confident degrees [30]. Regarding hesitant LNV environment, similarity measures between hesitant LNVs were presented by the least common multiple cardinality and applied to hesitant linguistic neutrosophic DM problems [31]. By the hybrid form of LNV [25] and linguistic cubic numbers [20], linguistic neutrosophic cubic numbers and their aggregation operators were introduced for linguistic neutrosophic cubic DM problems [32,33].

However, the various linguistic concepts are all described in a unique universal set, and then in some decision situations there exist the assessment problems of alternatives over two-dimensional universal sets (TDUSs). For example, suppose a person would like to purchase a house in a group of four houses (a set of four alternatives $H=\left\{H_{1}, H_{2}, H_{3}, H_{4}\right\}$ ). In his/her attractive evaluation of houses, the price $\left(x_{1}\right)$, environment $\left(x_{2}\right)$, and traffic $\left(x_{3}\right)$ of the four houses are considered as a universal set $X=\left\{x_{1}, x_{2}, x_{3}\right\}$, and selecting two cities $c_{1}$ and $c_{2}$ are considered as another universal set $C=\left\{c_{1}, c_{2}\right\}$. Obviously, the above various linguistic arguments cannot represent such an assessment problem for each alternative $H_{j}(j=1,2,3,4)$ over the TDUSs $X=\left\{x_{1}, x_{2}, x_{3}\right\}$ and $C=\left\{c_{1}, c_{2}\right\}$ in linguistic DM setting. Then, a Q-neutrosophic set and a Q-neutrosophic soft set were put forward regarding TDUSs and applied to Q-neutrosophic soft DM problems [34]. Although they can express and handle the assessment problems with TDUSs in neutrosophic DM environments, they cannot carry out linguistic neutrosophic DM problems over TDUSs. To solve this problem, this study presents a Q-linguistic neutrosophic variable set (Q-LNVS) for the first time to express the linguistic evaluation problems of the truth, falsity, and indeterminacy over TDUSs from the predefined linguistic term set (LTS). It then puts forward the vector similarity measures of Q-LNVSs, including the cosine, Dice, and Jaccard measures of Q-LNVSs, and then establishes a multi-attribute DM approach of Q-LNVSs by the vector similarity measures of Q-LNVs to solve linguistic neutrosophic DM problems along with TDUSs. It is obvious that the proposed DM approach shows the advantage of carrying out the linguistic neutrosophic DM problems regarding TDUSs, which existing linguistic neutrosophic DM approaches [25-28] and Q-linguistic neutrosophic soft DM approaches [34] cannot solve.

The framework of this study is organized below. The second section proposes Q-LNVSs and vector similarity measures between Q-LNVSs, including the cosine, Dice, and Jaccard measures of Q-LNVSs. The third section develops a multi-attribute DM approach of Q-LNVSs by using the vector similarity measures in Q-linguistic neutrosophic setting. An illustrative example and its sensitivity analysis to weights are presented in the fourth section. The last section contains conclusions and future study.

\section{Vector Similarity Measures of Q-LNVSs}

First, we present the concept of Q-LNVS to depict a linguistic neutrosophic evaluation problem by the truth, falsity, and indeterminacy linguistic arguments over TDUSs in linguistic setting. 
Definition 1. Set $X=\left\{x_{1}, x_{2}, \ldots, x_{n}\right\}$ and $Q=\left\{q_{1}, q_{2}, \ldots, q_{m}\right\}$ as TDUSs and let LTS be $S=\left\{s_{l} \mid l \in[0, g]\right\}$, where $g+1$ is an odd cardinality. Then a Q-LNVS L in X and $Q$ is defined by the following form:

$$
L=\left\{\begin{array}{l}
\left\langle\left(x_{i}, q_{j}\right), s_{t}\left(x_{i}, q_{j}\right), s_{u}\left(x_{i}, q_{j}\right), s_{v}\left(x_{i}, q_{j}\right)\right\rangle \mid x_{i} \in X, q_{j} \in Q, \\
s_{t}\left(x_{i}, q_{j}\right), s_{i}\left(x_{i}, q_{j}\right), s_{f}\left(x_{i}, q_{j}\right) \in S, j=1,2, \ldots, m ; i=1,2, \ldots, n
\end{array}\right\}
$$

where $s_{t}\left(x_{i}, q_{j}\right), s_{u}\left(x_{i}, q_{j}\right), s_{v}\left(x_{i}, q_{j}\right)$ are the truth, indeterminacy, and falsity LVs, respectively, in TDUSs for $t$, $u, v \in[0, g]$.

Then, the basic linguistic element $\left\langle\left(x_{i}, q_{j}\right), s_{t}\left(x_{i}, q_{j}\right), s_{u}\left(x_{i}, q_{j}\right), s_{v}\left(x_{i}, q_{j}\right)\right\rangle$ in $L$ is simply denoted by $l_{i j}=\left\langle\left(x_{i}, q_{j}\right), s_{t_{i j}}, s_{u_{i j}}, s_{v_{i j}}\right\rangle$, which is called a Q-linguistic neutrosophic element (Q-LNE).

Example 1. Suppose a person would like to buy a house from a city. There is a set of two potential houses $H=\left\{L_{1}, L_{2}\right\}$ in two cities. Then, set their price $\left(x_{1}\right)$, environment $\left(x_{2}\right)$, and traffic $\left(x_{3}\right)$ as a universal set $X=\left\{x_{1}, x_{2}, x_{3}\right\}$ and set the two cities as another universal set $Q=\left\{q_{1}, q_{2}\right\}$. Based on the predefined LTS $S=\left\{s_{0}=\right.$ extremely low, $s_{1}=$ very low, $s_{2}=$ low, $s_{3}=$ slightly low, $s_{4}=$ medium, $s_{5}=$ slightly high, $s_{6}=$ high, $s_{7}=$ very high $s_{8}=$ extremely high $\}$, the two $Q-L N E$ sets obtained from $S$ are given as follows:

$$
\begin{gathered}
L_{1}=\left\{<\left(x_{1}, q_{1}\right), s_{6}, s_{1}, s_{2}>,<\left(x_{1}, q_{2}\right), s_{5}, s_{2}, s_{3}>,<\left(x_{2}, q_{1}\right), s_{4}, s_{3}, s_{2}>,<\left(x_{2}, q_{2}\right), s_{7}, s_{1}, s_{3}>,\right. \\
\left.<\left(x_{3}, q_{1}\right), s_{6}, s_{2}, s_{1}>,<\left(x_{3}, q_{2}\right), s_{6}, s_{1}, s_{1}>\right\}, \\
L_{2}=\left\{<\left(x_{1}, q_{1}\right), s_{3}, s_{4}, s_{5}>,<\left(x_{1}, q_{2}\right), s_{4}, s_{2}, s_{1}>,<\left(x_{2}, q_{1}\right), s_{4}, s_{2}, s_{2}>,<\left(x_{2}, q_{2}\right), s_{5}, s_{1}, s_{2}>,\right. \\
\left.<\left(x_{3}, q_{1}\right), s_{5}, s_{2}, s_{1}>,<\left(x_{3}, q_{2}\right), s_{6}, s_{3}, s_{2}>\right\} .
\end{gathered}
$$

In the following, we give the vector similarity measures between Q-LNVSs, including the cosine, Dice, and Jaccard measures of Q-LNVSs.

Definition 2. Let TDUSs be $X=\left\{x_{1}, x_{2}, \ldots, x_{n}\right\}$ and $Q=\left\{q_{1}, q_{2}, \ldots, q_{p}\right\}$ and let $l_{i j}^{1}=\left\langle\left(x_{i}, q_{j}\right), s_{t_{i j}^{1}}, s_{u_{i j}^{1}}, s_{v_{i j}^{1}}\right\rangle$ and $l_{i j}^{2}=\left\langle\left(x_{i}, q_{j}\right), s_{t_{i j}^{2}}, s_{u_{i j}^{2}}, s_{v_{i j}^{2}}\right\rangle(j=1,2, \ldots, p ; i=1,2, \ldots, n)$ be two groups of Q-LNEs in two Q-LNVSs $L_{1}$ and $L_{2}$ regarding the LTS $S=\left\{s_{l} \mid l \in[0, g]\right\}$. Then, the cosine, Dice, and Jaccard measures of the $Q$-LNVSS $L_{1}$ and $L_{2}$ are defined, respectively, as follows:

$$
\begin{aligned}
& C\left(L_{1}, L_{2}\right)=s \frac{8 \Sigma_{j=1}^{p} \sum_{i=1}^{n}\left(\left(_{i j}^{1} t_{i j}^{2}+u_{i j}^{1} v_{i j}^{2}+v_{i j}^{1} v_{i j}^{2}\right)\right.}{\sqrt{\sum_{j=1}^{p} \sum_{i=1}^{n}\left[\left(t_{i j}^{1}\right)^{2}+\left(u_{i j}^{1}\right)^{2}+\left(v_{i j}^{1}\right)^{2}\right] \times \sqrt{\sum_{j=1}^{p} \sum_{i=1}^{n}\left[\left(t_{i j}^{2}\right)^{2}+\left(u_{i j}^{2}\right)^{2}+\left(v_{i j}^{2}\right)^{2}\right]}}}, \\
& D\left(L_{1}, L_{2}\right)=s \frac{2 g \Sigma_{j=1}^{p} \sum_{i=1}^{n}\left(t_{i j}^{1} t_{i j}^{2}+u_{i j}^{1} v_{i j}^{2}+v_{i j}^{1} v_{i j}^{2}\right)}{\sum_{j=1}^{p} \sum_{i=1}^{n}\left[\left(t_{i j}^{1}\right)^{2}+\left(u_{i j}^{1}\right)^{2}+\left(v_{i j}^{1}\right)^{2}\right)^{2}+\sum_{j=1}^{p} \sum_{i=1}^{n}\left[\left(t_{i j}^{2}\right)^{2}+\left(u_{i j}^{2}\right)^{2}+\left(v_{i j}^{2}\right)^{2}\right]}, \\
& J\left(L_{1}, L_{2}\right)=s \frac{g \sum_{j=1}^{p} \sum_{i=1}^{n}\left(t_{i j}^{1} t_{i j}^{2}+u_{i j}^{1} v_{i j}^{2}+v_{i j}^{1} v_{i j}^{2}\right)}{\sum_{j=1}^{p} \sum_{i=1}^{n}\left[\left(t_{i j}^{1}\right)^{2}+\left(u_{i j}^{1}\right)^{2}+\left(v_{i j}^{1}\right)^{2}\right]+\sum_{j=1}^{p} \sum_{i=1}^{n}\left[\left(t_{i j}^{2}\right)^{2}+\left(u_{i j}^{2}\right)^{2}+\left(v_{i j}^{2}\right)^{2}\right]-\sum_{j=1}^{p} \sum_{i=1}^{n}\left(t_{i j}^{1} t_{i j}^{2}+u_{i j}^{1} v_{i j}^{2}+v_{i j}^{1} v_{i j}^{2}\right)} .
\end{aligned}
$$

Obviously, the above cosine, Dice, and Jaccard measures satisfy the following properties:

(1) $s_{0} \leq C\left(L_{1}, L_{2}\right), D\left(L_{1}, L_{2}\right), J\left(L_{1}, L_{2}\right) \leq s_{g}$;

(2) $C\left(L_{1}, L_{2}\right)=C\left(L_{2}, L_{1}\right), D\left(L_{1}, L_{2}\right)=D\left(L_{2}, L_{1}\right), J\left(L_{1}, L_{2}\right)=J\left(L_{2}, L_{1}\right)$;

(3) $C\left(L_{1}, L_{2}\right)=D\left(L_{1}, L_{2}\right)=J\left(L_{1}, L_{2}\right)=s_{g}$ if and only if $L_{1}=L_{2}$.

When the importance of elements $x_{i}(i=1,2, \ldots, n)$ and $q_{j}(j=1,2, \ldots, p)$ is taken into account, the weight vectors corresponding to $X=\left\{x_{1}, x_{2}, \ldots, x_{n}\right\}$ and $Q=\left\{q_{1}, q_{2}, \ldots, q_{p}\right\}$ are given 
as $w=\left\{w_{1}, w_{2}, \ldots, w_{n}\right\}$ and $w=\left\{w_{1}, w_{2}, \ldots, w_{p}\right\}$, respectively. Thus, the weighted cosine, Dice, and Jaccard measures of $L_{1}$ and $L_{2}$ can be presented, respectively, as follows:

$$
\begin{aligned}
& C_{w}\left(L_{1}, L_{2}\right)=s \frac{8 \Sigma_{j=1}^{p} \omega_{j} \sum_{i=1}^{n} w_{i}\left(t_{i j}^{1} t_{i j}^{2}+u_{i j}^{1} v_{i j}^{2}+v_{i j}^{1} v_{i j}^{2}\right)}{\sqrt{\sum_{j=1}^{p} \omega_{j} \sum_{i=1}^{n} w_{i}\left[\left(t_{i j}^{1}\right)^{2}+\left(u_{i j}^{1}\right)^{2}+\left(v_{i j}^{1}\right)^{2}\right] \times \sqrt{\sum_{j=1}^{p} \omega_{j} \sum_{i=1}^{n} w_{i}\left[\left(t_{i j}^{2}\right)^{2}+\left(u_{i j}^{2}\right)^{2}+\left(v_{i j}^{2}\right)^{2}\right]}}}, \\
& D_{w}\left(L_{1}, L_{2}\right)=s \frac{2 g \Sigma_{j=1}^{p} \omega_{j} \sum_{i=1}^{n} w_{i}\left(t_{i j}^{1} t_{i j}^{2}+u_{i j}^{1} v_{i j}^{2}+v_{i j}^{1} v_{i j}^{2}\right)}{\sum_{j=1}^{p} \omega_{j} \sum_{i=1}^{n} w_{i}\left[\left(t_{i j}^{1}\right)^{2}+\left(u_{i j}^{1}\right)^{2}+\left(v_{i j}^{1}\right)^{2}\right)^{2}+\sum_{j=1}^{p} \omega_{j} \sum_{i=1}^{n} w_{i}\left[\left(t_{i j}^{2}\right)^{2}+\left(u_{i j}^{2}\right)^{2}+\left(v_{i j}^{2}\right)^{2}\right]}, \\
& J_{w}\left(L_{1}, L_{2}\right)=s \frac{g \sum_{j=1}^{p} \omega_{j} \sum_{i=1}^{n} w_{i}\left(t_{i j}^{1} t_{i j}^{2}+u_{i j}^{1} v_{i j}^{2}+v_{i j}^{1} v_{i j}^{2}\right)}{\sum_{j=1}^{p} \omega_{j} \sum_{i=1}^{n} w_{i}\left[\left(t_{i j}^{1}\right)^{2}+\left(u_{i j}^{1}\right)^{2}+\left(v_{i j}^{1}\right)^{2}\right]+\sum_{j=1}^{p} \omega_{j} \sum_{i=1}^{n} w_{i}\left[\left(t_{i j}^{2}\right)^{2}+\left(u_{i j}^{2}\right)^{2}+\left(v_{i j}^{2}\right)^{2}\right]-\sum_{j=1}^{p} \omega_{j} \sum_{i=1}^{n} w_{i}\left(t_{i j} t_{i j}^{2}+u_{i j}^{1} v_{i j}^{2}+v_{i j}^{1} v_{i j}^{2}\right)} .
\end{aligned}
$$

Example 2. Let us consider two Q-LNE sets $\left.L_{1}=\left\{<\left(x_{1}, q_{1}\right), s_{6}, s_{1}, s_{2}\right\rangle,<\left(x_{1}, q_{2}\right), s_{5}, s_{2}, s_{3}\right\rangle,<\left(x_{2}, q_{1}\right), s_{4}, s_{3}$, $\left.\left.\left.s_{2}\right\rangle,\left\langle\left(x_{2}, q_{2}\right), s_{7}, s_{1}, s_{3}\right\rangle,\left\langle\left(x_{3}, q_{1}\right), s_{6}, s_{2}, s_{1}\right\rangle,<\left(x_{3}, q_{2}\right), s_{6}, s_{1}, s_{1}\right\rangle\right\}$ and $L_{2}=\left\{<\left(x_{1}, q_{1}\right), s_{3}, s_{4}, s_{5}\right\rangle,<\left(x_{1}, q_{2}\right)$, $\left.\left.\left.s_{4}, s_{2}, s_{1}\right\rangle,<\left(x_{2}, q_{1}\right), s_{4}, s_{2}, s_{2}>,<\left(x_{2}, q_{2}\right), s_{5}, s_{1}, s_{2}\right\rangle,\left\langle\left(x_{3}, q_{1}\right), s_{5}, s_{2}, s_{1}\right\rangle,<\left(x_{3}, q_{2}\right), s_{6}, s_{3}, s_{2}>\right\}$ in the LTS $S=\left\{s_{0}, s_{1}, s_{2}, \ldots, s_{8}\right\}$ with $g=8$ and the TDUSs $X=\left\{x_{1}, x_{2}, x_{3}\right\}$ and $Q=\left\{q_{1}, q_{2}\right\}$. Suppose the weight vectors for $X=\left\{x_{1}, x_{2}, x_{3}\right\}$ and $Q=\left\{q_{1}, q_{2}\right\}$ are given as $w=(0.4,0.25,0.35)$ and $\omega=(0.4,0.6)$, respectively. Then, we compute the measure values of $C_{w}\left(L_{1}, L_{2}\right), D_{w}\left(L_{1}, L_{2}\right), J_{w}\left(L_{1}, L_{2}\right)$.

By using Equations (4)-(6), their calculational processes are shown as follows:

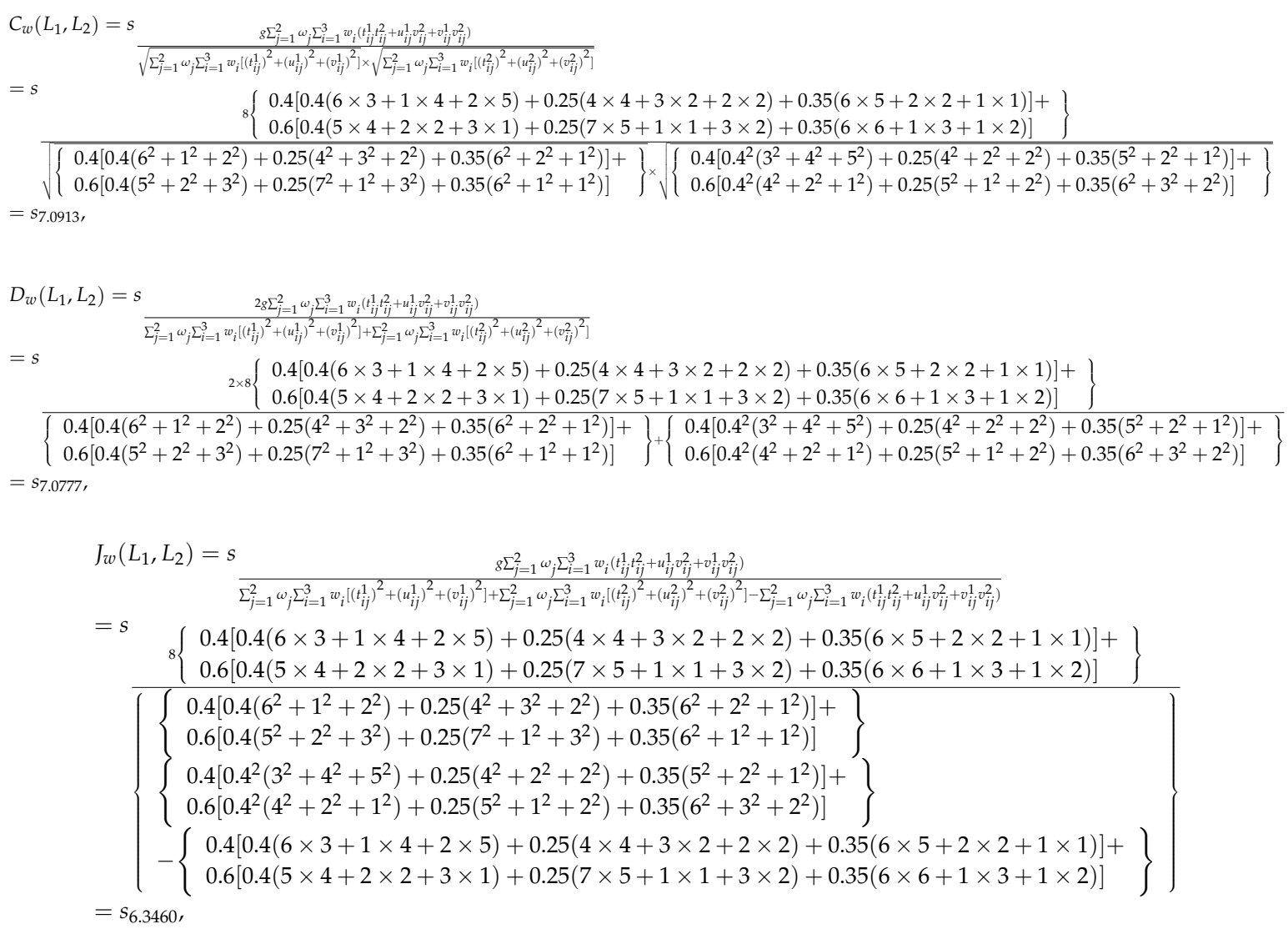

Obviously, the above vector measure values still belong to the LTS $S$.

\section{DM Approach Based on the Vector Similarity Measures}

This section proposes a Q-linguistic neutrosophic multi-attribute DM approach based on the Dice, cosine, and Jaccard measures (the three vector measures) of Q-LNVSs in Q-LNVS setting. 
Suppose there is a multi-attribute DM problem, in which $L=\left\{L_{1}, L_{2}, \ldots, L_{m}\right\}$ is denoted by a set of $m$ alternatives. Then, TDUSs (two kinds of attribute sets) are specified as $X=\left\{x_{1}, x_{2}, \ldots, x_{n}\right\}$ and $Q=\left\{q_{1}, q_{2}, \ldots, q_{p}\right\}$, respectively, and then their corresponding weigh vectors are given as $\boldsymbol{w}=\left(w_{1}, w_{2}, \ldots, w_{n}\right)$ and $\boldsymbol{w}=\left(w_{1}, w_{2}, \ldots, w_{p}\right)$. Whereas, a decision maker is required to assess the alternatives $L_{k}(k=1,2, \ldots, m)$ on the attributes $x_{i}(i=1,2, \ldots, n)$ and $q_{j}(j=1,2, \ldots, p)$ by Q-LNEs regarding the given LTS $S=\left\{s_{l} \mid l \in[0, g]\right\}$ with the odd cardinality $g+1$. In the assessment process, the decision maker gives the truth, falsity, and indeterminacy linguistic values for $x_{i}$ and $q_{j}$ on an alternative $L_{k}$ by corresponding linguistic terms in $S$, which are constructed as a Q-LNE $l_{i j}^{k}=\left\langle\left(x_{i}, q_{j}\right), s_{t_{i j}^{k}}, s_{u_{i j}^{k}}, s_{v_{i j}^{k}}\right\rangle(j=1,2, \ldots, p ; i=1,2, \ldots, n ; k=1,2, \ldots, m)$. Hence, all the Q-LNEs provided by the decision maker can be composed of a decision matrix of Q-LNEs $L=\left(l_{i j}^{k}\right)_{m \times n q}$.

Thus, the proposed DM method using the vector similarity measures of Q-LNVSs is applied to the multi-attribute DM problem with Q-LNVS information. Whereas, the DM steps are depicted in detail below:

Step 1: Since $l_{i j}^{*}=\left\langle\left(x_{i}, q_{j}\right), s_{t_{i j}^{*}}, s_{u_{i j}^{*}}, s_{v_{i j}^{*}}\right\rangle=\left\langle\left\langle\left(x_{i}, q_{j}\right), \max _{k}\left(s_{t_{i j}^{k}}\right), \min _{k}\left(s_{u_{i j}^{k}}\right), \min _{k}\left(s_{v_{i j}^{k}}\right)\right\rangle\right\rangle$ is an ideal Q-LNE as the best Q-LNE, we can establish the following ideal Q-LNVS:

$$
L^{*}=\left\{\left\langle\left(x_{i}, q_{j}\right), s_{t_{i j}^{*}}, s_{u_{i j}^{*}}, s_{v_{i j}^{*}}\right\rangle \mid x_{i} \in X, q_{j} \in Q, k=1,2, \ldots, m, j=1,2, \ldots, p, i=1,2, \ldots, n\right\} .
$$

Step 2: By applying Equations (4)-(6), the cosine/Dice/Jaccard measure between $L_{k}(k=1,2, \ldots, m)$ and $L^{*}$ is given by using the following formula:

$$
C_{w}\left(L_{k}, L^{*}\right)=s \frac{s \Sigma_{j=1}^{p} \omega_{j} \sum_{i=1}^{n} w_{i}\left(t_{i j}^{k} t_{i j}^{*}+u_{i j}^{k} v_{i j}^{*}+v_{i j}^{k} v_{i j}^{*}\right)}{\sqrt{\sum_{j=1}^{p} \omega_{j} \sum_{i=1}^{n} w_{i}\left[\left(t_{i j}^{k}\right)^{2}+\left(u_{i j}^{k}\right)^{2}+\left(v_{i j}^{k}\right)^{2}\right] \times \sqrt{\sum_{j=1}^{p} \omega_{j} \sum_{i=1}^{n} w_{i}\left[\left(t_{i j}^{*}\right)^{2}+\left(u_{i j}^{*}\right)^{2}+\left(v_{i j}^{*}\right)^{2}\right]}}},
$$

or

$$
D_{w}\left(L_{k}, L^{*}\right)=s \frac{2 \times g \Sigma_{j=1}^{p} \omega_{j}^{2} \sum_{i=1}^{n} w_{i}^{2}\left(t_{i j}^{k} k_{i j}^{*}+u_{i j}^{k} v_{i j}^{*}+v_{i j}^{k} v_{i j}^{*}\right)}{\sum_{j=1}^{p} \omega_{j}^{2} \Sigma_{i=1}^{n} w_{i}^{2}\left[\left(t_{i j}^{k}\right)^{2}+\left(u_{i j}^{k}\right)^{2}+\left(v_{i j}^{k}\right)^{2}\right]+\sum_{j=1}^{p} \omega_{j}^{2} \sum_{i=1}^{n} w_{i}^{2}\left[\left(t_{i j}^{*}\right)^{2}+\left(u_{i j}^{*}\right)^{2}+\left(v_{i j}^{*}\right)^{2}\right]},
$$

or

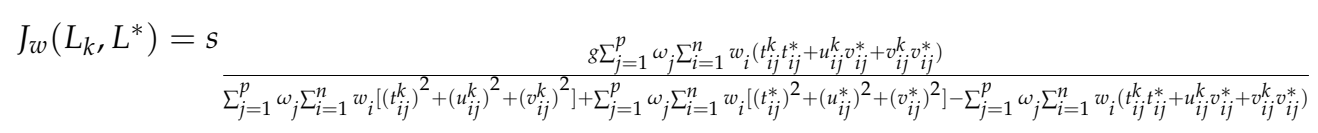

Step 3: According to the linguistic values of the vector similarity measures, the alternatives are ranked and the best alternative $L_{k^{*}}$ is chosen regarding the biggest linguistic value for $X$ and $Q$.

Step 4: Based on $x_{j} \in X(j=1,2, \ldots, p)$ or $q_{i} \in Q(i=1,2, \ldots, n)$, we need to calculate the measure values between $L_{k^{*}}\left(x_{i}, q_{j}\right)$ and $L^{*}\left(x_{i}, q_{j}\right)$ :

$$
C\left(L_{k^{*}}\left(x_{i}, q_{j}\right), L^{*}\left(x_{i}, q_{j}\right)\right)=s \frac{s \sum_{i=1}^{n}\left(t_{i j}^{k}+t_{i j}^{*}+u_{i j}^{k} j_{i j}^{*}+v_{i j}^{k} v_{i j}^{*}\right)}{\sqrt{\sum_{i=1}^{n}\left[\left(t_{i j}^{k}\right)^{2}+\left(u_{i j}^{k}\right)^{2}+\left(v_{i j}^{k}\right)^{2}\right] \times \sqrt{\Sigma_{i=1}^{n}\left[\left(t_{i j}^{*}\right)^{2}+\left(u_{i j}^{*}\right)^{2}+\left(v_{i j}^{*}\right)^{2}\right]}}} \text { for } j=1,2, \ldots, p,
$$

or

$$
\begin{aligned}
& C\left(L_{k^{*}}\left(x_{i}, q_{j}\right), L^{*}\left(x_{i}, q_{j}\right)\right)=s \frac{8 \Sigma_{j=1}^{p}\left(k_{i j}^{k} k_{i j}^{*}+u_{i j}^{k} v_{i j}^{*}+v_{i j}^{k} k_{i j}^{*}\right)}{\sqrt{\left.\sum_{j=1}^{p}\left[\left(t_{i j}^{k}\right)^{2}+\left(u_{i j}^{k}\right)^{2}+\left(v_{i j}^{k}\right)^{k}\right)^{2}\right] \times \sqrt{\sum_{j=1}^{p}\left[\left(t_{i j}^{*}\right)^{2}+\left(u_{i j}^{*}\right)^{2}+\left(v_{i j}^{*}\right)^{2}\right]}}} \text { for } i=1,2, \ldots, n \text {; } \\
& D\left(L_{k^{*}}\left(x_{i}, q_{j}\right), L^{*}\left(x_{i}, q_{j}\right)\right)=s \frac{2 g \sum_{i=1}^{n}\left(t_{i j}^{k} j_{i j}^{*}+u_{i j}^{k} v_{i j}^{*}+v_{i j}^{k} v_{i j}^{*}\right)}{\sum_{i=1}^{n}\left[\left(t_{i j}^{k}\right)^{2}+\left(u_{i j}^{k}\right)^{2}+\left(v_{i j}^{k}\right)^{2}\right]+\sum_{i=1}^{n}\left[\left(t_{i j}^{*}\right)^{2}+\left(u_{i j}^{*}\right)^{2}+\left(v_{i j}^{*}\right)^{2}\right]} \quad \text { for } j=1,2, \ldots, p \text {, }
\end{aligned}
$$


or

$$
\begin{aligned}
& D\left(L_{k^{*}}\left(x_{i}, q_{j}\right), L^{*}\left(x_{i}, q_{j}\right)\right)=s \frac{2 g \sum_{j=1}^{p}\left(t_{i j}^{k} t_{i j}^{*}+u_{i j}^{k} v_{i j}^{*}+v_{i j}^{k} v_{i j}^{*}\right)}{\sum_{j=1}^{p}\left[\left(t_{i j}^{k}\right)^{2}+\left(u_{i j}^{k}\right)^{2}+\left(v_{i j}^{k}\right)^{2}\right]+\sum_{j=1}^{p}\left[\left(t_{i j}^{*}\right)^{2}+\left(u_{i j}^{*}\right)^{2}+\left(v_{i j}^{*}\right)^{2}\right]} \quad \text { for } i=1,2, \ldots, n ; \\
& J\left(L_{k^{*}}\left(x_{i}, q_{j}\right), L^{*}\left(x_{i}, q_{j}\right)\right)=s \frac{8 \sum_{i=1}^{n}\left(t_{i j}^{k} t_{i j}^{*}+u_{i j}^{k} v_{i j}^{*}+v_{i j}^{k} v_{i j}^{*}\right)}{\sum_{i=1}^{n}\left[\left(t_{i j}^{k}\right)^{2}+\left(u_{i j}^{k}\right)^{2}+\left(v_{i j}^{k}\right)^{2}\right]+\sum_{i=1}^{n}\left[\left(t_{i j}^{*}\right)^{2}+\left(u_{i j}^{*}\right)^{2}+\left(v_{i j}^{*}\right)^{2}\right]-\sum_{i=1}^{n}\left(t_{i j}^{k} t_{i j}^{*}+u_{i j}^{k} v_{i j}^{*}+v_{i j}^{k} v_{i j}^{*}\right)} \quad \text { for } j=1,2, \ldots, p,
\end{aligned}
$$

or

$$
J\left(L_{k^{*}}\left(x_{i}, q_{j}\right), L^{*}\left(x_{i}, q_{j}\right)\right)=s \frac{g \sum_{j=1}^{p}\left(t_{i j}^{k} t_{i j}^{*}+u_{i j}^{k} v_{i j}^{*}+v_{i j}^{k} v_{i j}^{*}\right)}{\sum_{j=1}^{p}\left[\left(t_{i j}^{k}\right)^{2}+\left(u_{i j}^{k}\right)^{2}+\left(v_{i j}^{k}\right)^{2}\right]+\sum_{j=1}^{p}\left[\left(t_{i j}^{*}\right)^{2}+\left(u_{i j}^{*}\right)^{2}+\left(v_{i j}^{*}\right)^{2}\right]-\sum_{j=1}^{p}\left(t_{i j}^{k} t_{i j}^{*}+u_{i j}^{k} v_{i j}^{*}+v_{i j}^{k} v_{i j}^{*}\right)} \quad \text { for } i=1,2, \ldots, n \text {. }
$$

Step 5: According to the linguistic values of $C\left(L_{k^{*}}\left(x_{i}, q_{j}\right), L^{*}\left(x_{i}, q_{j}\right)\right)$ or $D\left(L_{k^{*}}\left(x_{i}, q_{j}\right), L^{*}\left(x_{i}, q_{j}\right)\right)$ or $J\left(L_{k^{*}}\left(x_{i}, q_{j}\right), L^{*}\left(x_{i}, q_{j}\right)\right)$ for $X$ or $Q$ (depending on some actual situation), we can determine the best one $x_{i *}$ or $q_{j *}$ corresponding to the biggest linguistic value.

Step 6: End.

\section{Illustrative Example and Sensitivity Analysis to Weights}

\subsection{Illustrative Example}

Suppose a person would like to buy a house in one of two cities. There are four potential houses (alternatives) of $L_{k}(k=1,2,3,4)$ in two cities. Then, set their price $\left(x_{1}\right)$, environment $\left(x_{2}\right)$, and traffic $\left(x_{3}\right)$ as a universal set $X=\left\{x_{1}, x_{2}, x_{3}\right\}$ and set the two cities as another universal set $Q=\left\{q_{1}, q_{2}\right\}$. Thus, the Q-LNEs can indicate the influence of both the three attributes of houses and the two cities on his/her buying attractive degree of a house. Herewith, the two weigh vectors of $X$ and $Q$ are given as $w=(0.4,0.25,0.35)$ and $w=(0.4,0.6)$, respectively. Whereas, the alternative $L_{k}(k=1,2,3,4)$ are assessed over the TDUSs $X=\left\{x_{1}, x_{2}, x_{3}\right\}$ and $Q=\left\{q_{1}, q_{2}\right\}$ from the given LTS $S=\left\{s_{0}=\right.$ extremely low, $s_{1}=$ very low,$s_{2}=$ low, $s_{3}=$ slightly low, $s_{4}=$ medium, $s_{5}=$ slightly high, $s_{6}=$ high, $s_{7}=$ very high, $s_{8}=$ extremely high $\}$ with $g=8$. In the assessment process, the decision maker/buyer can give the truth, indeterminacy, and falsity values for $x_{i}$ and $q_{j}$ on an alternative $L_{k}$ by corresponding linguistic terms in $S$, and then establish Q-LNEs $l_{i j}^{k}=\left\langle\left(x_{i}, q_{j}\right), s_{t_{i j}^{k}}, s_{u_{i j}^{k}}, s_{v_{i j}^{k}}\right\rangle(j=1,2 ; i=1,2,3 ; k=1,2,3,4)$, which are constructed as the DM matrix of Q-LNEs:

$L=\begin{aligned} & L_{1} \\ & L_{2} \\ & L_{3} \\ & L_{4}\end{aligned}\left[\begin{array}{lllllll}\left\langle\left(x_{1}, q_{1}\right), s_{6}, s_{2}, s_{1}\right\rangle & \left\langle\left(x_{1}, q_{2}\right), s_{7}, s_{2}, s_{3}\right\rangle & \left\langle\left(x_{2}, q_{1}\right), s_{5}, s_{2}, s_{1}\right\rangle & \left\langle\left(x_{2}, q_{2}\right), s_{4}, s_{1}, s_{1}\right\rangle & \left\langle\left(x_{3}, q_{1}\right), s_{5}, s_{2}, s_{2}\right\rangle & \left\langle\left(x_{3}, q_{2}\right), s_{7}, s_{2}, s_{2}\right\rangle \\ \left\langle\left(x_{1}, q_{1}\right), s_{6}, s_{1}, s_{2}\right\rangle & \left\langle\left(x_{1}, q_{2}\right), s_{7}\right\rangle & \left\langle\left(x_{1}, s_{1}\right\rangle\right. & \left\langle\left(x_{2}, q_{1}\right), s_{6}, s_{1}, s_{1}\right\rangle & \left\langle\left(x_{2}, q_{2}\right), s_{5}, s_{1}, s_{2}\right\rangle & \left\langle\left(x_{3}, q_{1}\right), s_{6}, s_{1}, s_{3}\right\rangle & \left\langle\left(x_{3}, q_{2}\right), s_{7}, s_{2}, s_{1}\right\rangle \\ \left\langle\left(x_{1}, q_{1}\right), s_{5}, s_{1}, s_{1}\right\rangle & \left.\left\langle\left(x_{1}, q_{2}\right), s_{4}\right), s_{6}, s_{3}, s_{5}\right\rangle & \left\langle\left(x_{2}, q_{2}\right), s_{5}, s_{2}, s_{2}\right\rangle & \left\langle\left(x_{3}, q_{1}\right), s_{4}, s_{2}, s_{2}\right\rangle & \left\langle\left(x_{3}, q_{2}\right), s_{5}, s_{2}, s_{3}\right\rangle \\ & \left\langle\left(x_{2}, q_{1}\right), s_{5}, s_{3}, s_{3}\right\rangle & \left\langle\left(x_{2}, q_{2}\right), s_{6}, s_{2}, s_{4}\right\rangle & \left\langle\left(x_{3}, q_{1}\right), s_{5}, s_{1}, s_{1}\right\rangle & \left\langle\left(x_{3}, q_{2}\right), s_{6}, s_{2}, s_{3}\right\rangle\end{array}\right]$

Thus, the proposed multi-attribute DM approach can be used for this Q-linguistic neutrosophic DM problem. The DM steps are depicted below:

Firstly, we establish the ideal alternative from the DM matrix $L$ by the ideal Q-LNE set:

$L^{*}=\left\{\left\langle\left(x_{1}, q_{1}\right), s_{6}, s_{1}, s_{1}\right\rangle,\left\langle\left(x_{1}, q_{2}\right), s_{7}, s_{1}, s_{1}\right\rangle,\left\langle\left(x_{2}, q_{1}\right), s_{6}, s_{1}, s_{1}\right\rangle,\left\langle\left(x_{2}, q_{2}\right), s_{6}, s_{1}, s_{1}\right\rangle,\left\langle\left(x_{3}, q_{1}\right), s_{6}, s_{1}, s_{1}\right\rangle,\left\langle\left(x_{3}, q_{2}\right), s_{7}, s_{2}, s_{1}\right\rangle\right\}$. Table 1.

Then, by Equations (8)-(10), the measure results and ranking of the four alternatives are given in 
Table 1. Measure results and ranking of the four alternatives.

\begin{tabular}{ccc}
\hline Measure Method & Measure Value between $\boldsymbol{L}_{\boldsymbol{k}}(\boldsymbol{k}=\mathbf{1}, \mathbf{2 , 3}, \mathbf{4})$ and $\boldsymbol{L}^{*}$ & Ranking \\
\hline$C_{w}\left(L_{k}, L^{*}\right)$ & $s_{7.7472}, s_{7.9088}, s_{7.3465}, s_{7.2437}$ & $L_{2}>L_{1}>L_{3}>L_{4}$ \\
$D_{w}\left(L_{k}, L^{*}\right)$ & $s_{7.7470}, s_{7.9087}, s_{7.3207}, s_{7.2387}$ & $L_{2}>L_{1}>L_{3}>L_{4}$ \\
$J_{w}\left(L_{k}, L^{*}\right)$ & $s_{7.5095}, s_{7.8194}, s_{6.7478}, s_{6.6097}$ & $L_{2}>L_{1}>L_{3}>L_{4}$ \\
\hline
\end{tabular}

Based on Table 1, all the ranking orders are identical regarding the cosine, Dice, and Jaccard measures. Then, the best alternative is $L_{2}$.

Next, the measure values of Equations (11), (13), and (15) regarding $Q$, and the best city regarding $L_{2}$ are given in Table 2 .

Table 2. Measure results regarding $Q$ and the best city.

\begin{tabular}{ccc}
\hline Measure Method & Measure Result & The Best City \\
\hline$C\left(L_{2}\left(x_{i}, q_{1}\right), L^{*}\left(x_{i}, q_{1}\right)\right), C\left(L_{2}\left(x_{i}, q_{2}\right), L^{*}\left(x_{i}, q_{2}\right)\right)$ & $s_{7.8409}, s_{7.9457}$ & $q_{2}$ \\
$D\left(L_{2}\left(x_{i}, q_{1}\right), L^{*}\left(x_{i}, q_{1}\right)\right), D\left(L_{2}\left(x_{i}, q_{2}\right), L^{*}\left(x_{i}, q_{2}\right)\right)$ & $s_{7.8326}, s_{7.9424}$ & $q_{2}$ \\
$J\left(L_{2}\left(x_{i}, q_{1}\right), L^{*}\left(x_{i}, q_{1}\right)\right), J\left(L_{2}\left(x_{i}, q_{2}\right), L^{*}\left(x_{i}, q_{2}\right)\right)$ & $s_{7.6721}, s_{7.8857}$ & $q_{2}$ \\
\hline
\end{tabular}

Lastly, the results based on Table 2 indicate that the buyer should buy the house $L_{2}$ in the best city $q_{2}$.

\subsection{Sensitivity Analysis to Weights}

To indicate the influence of the weights on ranking orders in the illustrative example, we consider that the two weigh vectors of $X$ and $Q$ are given as $w=(1 / 3,1 / 3,1 / 3)$ and $w=(1 / 2,1 / 2)$, respectively, to analyze the sensitivity of the weights with respect to the ranking orders of the four alternatives. In this case, by Equations (8)-(10) the measure results and ranking of the four alternatives are indicated in Table 3.

Table 3. Measure results and ranking of the four alternatives with $w=(1 / 3,1 / 3,1 / 3)$ and $w=(1 / 2,1 / 2)$.

\begin{tabular}{ccc}
\hline Measure Method & Measure Value between $\boldsymbol{L}_{\boldsymbol{k}}(\boldsymbol{k}=\mathbf{1 , 2 , 3}, \mathbf{4})$ and $\boldsymbol{L}^{*}$ & Ranking \\
\hline$C_{w}\left(L_{k}, L^{*}\right)$ & $s_{7.7470}, s_{7.8918}, s_{7.3878}, s_{7.2922}$ & $L_{2}>L_{1}>L_{3}>L_{4}$ \\
$D_{w}\left(L_{k}, L^{*}\right)$ & $s_{7.7430}, s_{7.8917}, s_{7.3448}, s_{7.2892}$ & $L_{2}>L_{1}>L_{3}>L_{4}$ \\
$J_{w}\left(L_{k}, L^{*}\right)$ & $s_{7.5019}, s_{7.7863}, s_{6.7888}, s_{6.6944}$ & $L_{2}>L_{1}>L_{3}>L_{4}$ \\
\hline
\end{tabular}

In this case, there exists the same ranking order in Tables 1 and 3 regarding the cosine, Dice, and Jaccard measures. Then, the best alternative is sill $L_{2}$, which means the buyer should also buy house $L_{2}$ in the best city $q_{2}$ based on Table 2 . It is obvious that all the ranking orders imply the decision robustness based on the cosine, Dice, and Jaccard measures regarding the change of weights in this illustrative example, which also show no sensitivity of all the ranking orders with respect to the change of the weights. In the actual DM applications, however, one of three vector measures can be selected by decision makers' preference or actual requirements.

However, existing various linguistic neutrosophic DM approaches [25-28] cannot handle the DM problems in Q-LNVS setting; while our proposed DM method can carry out both the existing DM problems with LNV information [25-28] and the DM problems with Q-LNVS information, which shows its advantage in Q-LNVS setting because the LNV set is a special case of Q-LNVS under a universal set. Furthermore, the existing Q-neutrosophic soft DM approach [34] cannot deal with the DM problems with Q-LNVS information because the Q-neutrosophic soft set [34] cannot express Q-linguistic neutrosophic information. Hence, our proposed Q-linguistic neutrosophic DM method provides a new way for linguistic neutrosophic DM with TDUSs. 


\section{Conclusions}

This study presented the concept of Q-LNVS for the first time to describe the truth, falsity, and indeterminacy linguistic arguments in TDUSs, and then the cosine, Dice, and Jaccard measures of Q-LNVSs in vector space. Next, a Q-linguistic neutrosophic multi-attribute DM approach in Q-LNVS setting was established by using the cosine, Dice, and Jaccard measures of Q-LNVSs to solve linguistic neutrosophic DM problems regarding TDUSs. Lastly, the application of the developed DM approach was given by an illustrative example in Q-LNVS setting. The decision results show that the established multi-attribute DM approach of Q-LNVSs can solve linguistic neutrosophic DM problems regarding TDUSs (two-dimensional attribute sets) in Q-LNVS setting, which indicates its main advantage and contribution. Based on the first study, the three vector measures of Q-LNVSs will be further used for medical diagnosis, data mining, and clustering analysis for future research in Q-LNVS setting.

Author Contributions: J.Y. proposed the Q-LNVS concept and the vector similarity measures; Z.F. and W.C. gave the DM approach and the calculation and analysis; all the authors wrote the manuscript and revised the final version.

Funding: This paper was supported by the National Natural Science Foundation of China (Nos. 61703280, 51872186).

Conflicts of Interest: The authors declare no conflicts of interest.

\section{References}

1. Zadeh, L.A. The concept of a linguistic variable and its application to approximate reasoning Part I. Inf. Sci. 1975, 8, 199-249. [CrossRef]

2. Herrera, F.; Herrera-Viedma, E.; Verdegay, L. A model of consensus in group decision making under linguistic assessments. Fuzzy Sets Syst. 1996, 79, 73-87. [CrossRef]

3. Herrera, F.; Herrera-Viedma, E. Linguistic decision analysis: Steps for solving decision problems under linguistic information. Fuzzy Sets Syst. 2000, 115, 67-82. [CrossRef]

4. $\mathrm{Xu}, \mathrm{Z}$.S. A method based on linguistic aggregation operators for group decision making with linguistic preference relations. Inf. Sci. 2004, 166, 19-30. [CrossRef]

5. Xu, Z.S. A note on linguistic hybrid arithmetic averaging operator in multiple attribute group decision making with linguistic information. Group Decis. Negot. 2006, 15, 593-604. [CrossRef]

6. Merigó, J.M.; Casanovas, M.; Martínez, L. Linguistic aggregation operators for linguistic decision making based on the Dempster-Shafer theory of evidence. Int. J. Uncertain. Fuzz. Knowl.-Based Syst. 2010, 18, 287-304. [CrossRef]

7. Xu, Y.J.; Merigó, J.M.; Wang, H.M. Linguistic power aggregation operators and their application to multiple attribute group decision making. Appl. Math. Model. 2012, 36, 5427-5444. [CrossRef]

8. Merigó, J.M.; Casanovas, M.; Palacios-Marqués, D. Linguistic group decision making with induced aggregation operators and probabilistic information. Appl. Soft Comput. 2014, 24, 669-678. [CrossRef]

9. Merigó, J.M.; Palacios-Marqués, D.; Zeng, S.Z. Subjective and objective information in linguistic multi-criteria group decision making. Eur. J. Oper. Res. 2016, 248, 522-531. [CrossRef]

10. $\mathrm{Xu}, \mathrm{Z}$.S. Uncertain linguistic aggregation operators based approach to multiple attribute group decision making under uncertain linguistic environment. Inf. Sci. 2004, 168, 171-184. [CrossRef]

11. $\mathrm{Xu}, \mathrm{Z}$.S. Induced uncertain linguistic OWA operators applied to group decision making. Inf. Fusion 2006, 7, 231-238. [CrossRef]

12. Wei, G.W. Uncertain linguistic hybrid geometric mean operator and its application to group decision making under uncertain linguistic environment. Int. J. Uncertain. Fuzz. Knowl.-Based Syst. 2009, 17, 251-267. [CrossRef]

13. Park, J.H.; Gwak, M.G.; Kwun, Y.C. Uncertain linguistic harmonic mean operators and their applications to multiple attribute group decision making. Computing 2011, 93, 47. [CrossRef]

14. Wei, G.W.; Zhao, X.F.; Lin, R.; Wang, H.J. Uncertain linguistic Bonferroni mean operators and their application to multiple attribute decision making. Appl. Math. Model. 2013, 37, 5277-5285. [CrossRef]

15. Rodríguez, R.M.; Martínez, L.; Herrera, F. Hesitant fuzzy linguistic term sets for decision making. IEEE Trans. Fuzzy Syst. 2012, 20, 109-119. [CrossRef] 
16. Rodríguez, R.M.; MartíNez, L.; Herrera, F. A group decision making model dealing with comparative linguistic expressions based on hesitant fuzzy linguistic term sets. Inf. Sci. 2013, 241, 28-42. [CrossRef]

17. Lin, R.; Zhao, X.F.; Wei, G.W. Models for selecting an ERP system with hesitant fuzzy linguistic information. J. Intell. Fuzzy Syst. 2014, 26, 2155-2165.

18. Zhu, B.; Xu, Z. Consistency measures for hesitant fuzzy linguistic preference relations. IEEE Trans. Fuzzy Syst. 2014, 22, 35-45. [CrossRef]

19. Wang, J.Q.; Wu, J.T.; Wang, J.; Zhang, H.; Chen, X. Interval-valued hesitant fuzzy linguistic sets and their applications in multi-criteria decision-making problems. Inf. Sci. 2014, 288, 55-72. [CrossRef]

20. Ye, J. Multiple attribute decision-making method based on linguistic cubic variables. J. Intell. Fuzzy Syst. 2018, 34, 2351-2361. [CrossRef]

21. Lu, X.P.; Ye, J. Dombi aggregation operators of linguistic cubic variables for multiple attribute decision making. Information 2018, 9, 188. [CrossRef]

22. Ye, J.; Cui, W.H. Multiple attribute decision-making method using linguistic cubic hesitant variables. Algorithms 2018, 11, 135. [CrossRef]

23. Ye, J. Aggregation operators of neutrosophic linguistic numbers for multiple attribute group decision making. SpringerPlus 2016, 5, 1691. [CrossRef] [PubMed]

24. Ye, J. Multiple attribute decision-making methods based on expected value and similarity measure of hesitant neutrosophic linguistic numbers. Cogn. Comput. 2018, 10, 454-463. [CrossRef]

25. Fang, Z.B.; Ye, J. Multiple attribute group decision-making method based on linguistic neutrosophic numbers. Symmetry 2017, 9, 111. [CrossRef]

26. Fan, C.X.; Ye, J.; Hu, K.L.; Fan, E. Bonferroni mean operators of linguistic neutrosophic numbers and their multiple attribute group decision-making methods. Information 2017, 8, 107. [CrossRef]

27. Shi, L.L.; Ye, J. Cosine measures of linguistic neutrosophic numbers and their application in multiple attribute group decision-making. Information 2017, 8, 117. [CrossRef]

28. Shi, L.L.; Ye, J. Multiple attribute group decision-making method using correlation coefficients between linguistic neutrosophic numbers. J. Intell. Fuzzy Syst. 2018, 35, 917-925. [CrossRef]

29. Cui, W.H.; Ye, J.; Shi, L.L. Linguistic neutrosophic uncertain numbers and their multiple attribute group decision-making method. J. Intell. Fuzzy Syst. 2018. [CrossRef]

30. Ye, J.; Cui, W.H. Operations and aggregation methods of single-valued linguistic neutrosophic interval linguistic numbers and their decision making method. Information 2018, 9, 196. [CrossRef]

31. Cui, W.H.; Ye, J. Multiple-attribute decision-making method using similarity measures of hesitant linguistic neutrosophic numbers regarding least common multiple cardinality. Symmetry 2018, 10, 330. [CrossRef]

32. Ye, J. Linguistic neutrosophic cubic numbers and their multiple attribute decision-making method. Information 2017, 8, 110. [CrossRef]

33. Fan, C.X.; Ye, J. Heronian mean operator of linguistic neutrosophic cubic numbers and their multiple attribute decision-making methods. Math. Probl. Eng. 2018, 2018, 4158264. [CrossRef]

34. Qamar, M.A.; Hassan, N. Q-Neutrosophic soft relation and its application in decision making. Entropy 2018, 20, 172. [CrossRef]

(C) 2018 by the authors. Licensee MDPI, Basel, Switzerland. This article is an open access article distributed under the terms and conditions of the Creative Commons Attribution (CC BY) license (http://creativecommons.org/licenses/by/4.0/). 